\title{
Designing Atomic Edge Structures in 2D Transition Metal Dichalcogenides for Improved Catalytic Activity
}

Raymond Unocic ${ }^{1}$, Xiahan Sang ${ }^{2}$, Guoxiang $\mathrm{Hu}^{3}$, Victor Fung ${ }^{4}$, Matthew Boebinger ${ }^{1}$, Kai Xiao ${ }^{1}$ and Panchapakesan Ganesh ${ }^{4}$

${ }^{1}$ Oak Ridge National Laboratory, United States, ${ }^{2}$ Wuhan University of Technology, Wuhan, China (People's Republic), ${ }^{3}$ Queens College of the City University of New York, Queens, New York, United States, ${ }^{4}$ Oak Ridge National Laboratory, Oak Ridge, Tennessee, United States

Designing new materials for functional applications depends on our ability to understand and correlate the materials structure and chemistry to functional properties. This is even more important for twodimensional (2D) materials where the thickness is typically on the order of a single atom to a few-atomic layers; therefore, any structural or chemical modification at these length scales can have a profound effect on modifying the physical and chemical properties. 2D transition metal dichalcogenides (TMDs), such as $\mathrm{MoS}_{2}$, have emerged as a promising catalyst for the hydrogen evolution reaction (HER) with defects such as vacancies and edges linked to catalytically active sites as opposed to basal planes [1]. It is therefore important to develop controlled synthesis methods that promote the formation of atomic edge structures engineered for high catalytic HER activity.

To better understand the formation mechanisms of synthesizable atomic edge reconstructions, in situ scanning transmission electron microscopy (STEM) studies were performed to directly image and analyze the evolution of edge structures in a 2D TMD as a function of temperature and under controlled electron (e)-beam irradiation at atomic resolution [2]. In these studies, flakes of single layer, W-doped $\mathrm{MoSe}_{2}$ grown by chemical vapor deposition (CVD) were transferred to a MEMs-based heating microchip device for in situ heating experiments. Edge reconstructions that structurally transform by thermal and chemical driving forces, were characterized using time-resolved, atomic-resolution annular dark-field (ADF) STEM imaging. Figure 1 demonstrates a typical edge reconstruction where a zig zag (ZZ) Se-terminated edge structurally transforms first through a metastable Mo-rich ZZSe-GB4-Se (where GB4 refers to twin boundary) structure and then into the thermodynamically stable ZZSe-Mo-NW30 configuration (where NW30 refers to a nanowire rotated by $30^{\circ}$ ). In addition to this specific edge reconstruction, other thermodynamically stable, non-stoichiometric edges were formed that were linked to the evolving chemical potential resulting from the combined effects of thermal exposure, e-beam irradiation, and surface residue that locally alter the atomic structure. To computationally screen for thermodynamically stable and synthesizable atomic edge structures, density functional theory (DFT) and reactive force fields (ReaxFF) were used to calculate the formation enthalpy of a large number of atomic edge configurations [3]. The phase stability diagram, plotted as formation energy $\left(\mathrm{E}_{\mathrm{f}}\right)$ as a function of sulfur chemical potential $\left(\mu_{\mathrm{s}}\right)$, is shown in Figure 2a. The synthesis of more stable atomic edge structures can be attained through controlled molecular beam epitaxy [4] or controlled heat and e-beam irradiation (2). Based on these results, we show how non-stoichiometric engineered edges are catalytically active for the HER by calculating the Gibbs free energy $\left(\Delta \mathrm{G}_{\mathrm{H}}\right)$ of hydrogen adsorption (Figure $2 \mathrm{~b}$ ), which is a good descriptor for the HER [3,5]. The work presented here helps pave the way to design and engineer specific atomic edge structure in 2D TMDs for enhanced catalytic performance [6]. 

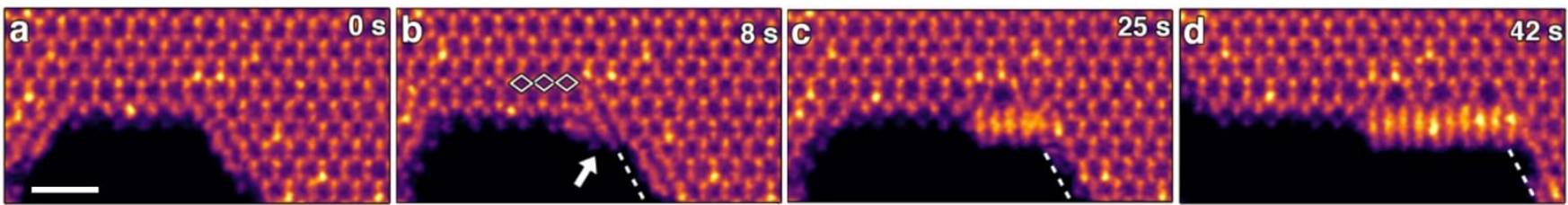

Figure 1. Figure 1. Time-resolved atomic-resolution ADF-STEM images showing atomic edge structure evolution from ZZSe-Se to ZZSe-Mo-NW30. Scale bar is $1 \mathrm{~nm}$ [2].
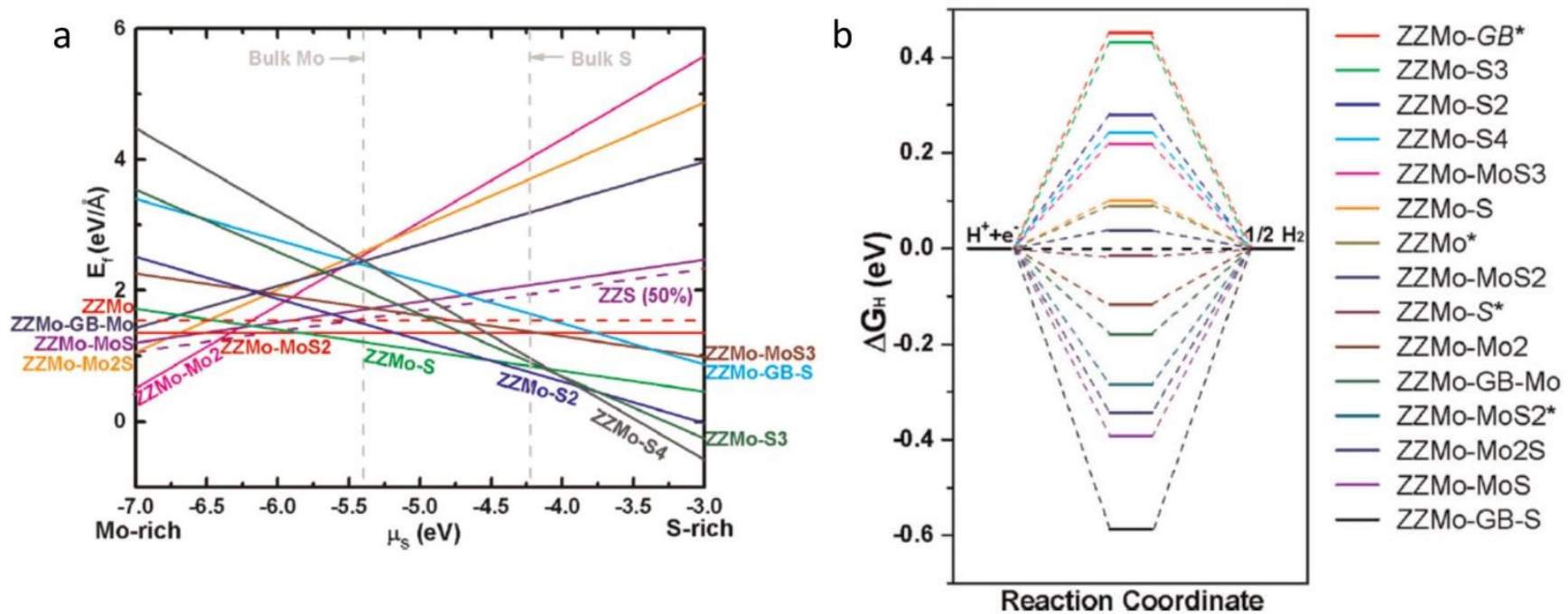

Figure 2. Figure 2. a) Plot of formation energies $\mathrm{Ef}(\mathrm{eV} / \AA)$ as a function of sulfur chemical potential $(\mu \mathrm{S})(\mathrm{eV})$ for several proposed atomic edge configurations. b) Calculated free energy diagram for the hydrogen evolution reaction for each of the predicted synthesizable edges [3].

\section{References}

[1] Jaramillo, T.F., et al. Science 317 (2007) p. 100

[2] Sang, X., et al., Nature Communications 9 (2018) p. 2051

[3] Hu, G., et al., npj Computational Materials 6 (2020) p.1

[4] Zhao, X., et al. Nano Lett, 18, 482-490, (2017), p. 482

[5] Hu, G., et al., Journal of Materials Chemistry A, 7, (2019) p.18357

[6] This research was supported by the Laboratory Directed Research and Development Program (LDRD) of Oak Ridge National Laboratory (ORNL), managed by UT Battelle, LLC, for the U.S. Department of Energy. A portion of this research was conducted at ORNL's Center for Nanophase Materials Sciences, which is a DOE Office of Science User Facility. This research used resources of the National Energy Research Scientific Computing Center, a DOE Office of Science User Facility supported by the Office of Science of the U.S. Department of Energy. 